American Journal of Applied Sciences 9 (4): 584-592, 2012

ISSN 1546-9239

(C) 2012 Science Publications

\title{
Some Applications of Spanning Trees in Complete and Complete Bipartite Graphs
}

\author{
${ }^{1,2}$ Daoud, S.N. \\ ${ }^{1}$ Department of Mathematics, \\ Faculty of Science, El-Minufiya University, Shebeen El-Kom, Egypt \\ ${ }^{2}$ Department of Applied Mathematics, \\ Faculty of Applied Science, Taibah University, Al-Madinah, KSA
}

\begin{abstract}
Problem statement: The number of spanning trees $\tau(\mathrm{G})$ in graphs (networks) is an important invariant, it is also an important measure of reliability of a network. Approach: Using linear algebra and matrix analysis techniques to evaluate the associated determinants. Results: In this study we derive simple formulas for the number of spanning trees of complete graph $K_{n}$ and complete bipartite graph $\mathrm{K}_{\mathrm{n}, \mathrm{m}}$ and some of their applications. A large number of theorems of number of the spanning trees of known operations on complete graph $K_{n}$ and complete bipartite graph $K_{n, m}$ are obtained. Conclusion: The evaluation of number of spanning trees is not only interesting from a mathematical (computational) perspective, but also, it is an important measure of reliability of a network and designing electrical circuits. Some computationally hard problems such as the travelling salesman problem can be solved approximately by using spanning trees. Due to the high dependence of the network design and reliability on the graph theory we introduced the following important theorems and lemmas and their proofs.
\end{abstract}

Key words: Complete graph, complete bipartite graph, spanning trees, Kirchhoff matrix, operations on graphs

\section{INTRODUCTION}

We consider finite undirected graph with no loops or multiple edges. Let $\mathrm{G}$ be such a graph on $\mathrm{n}$ vertices. A spanning tree for a graph $G$ is a subgraph of $G$ that is a tree and contains all vertices of $G$. There are many situation in which good spanning trees must be found. Whenever one wants to find a simple, cheap, yet efficient way to connect a set of terminals, be they computers, telephones, factories, or cities, a solution is normally one kind of spanning trees. Spanning trees prove important for several reasons: They create a spare subgraph that reflects a lot about the original graph, they play an important role to designing efficient routing algorithms, some computationally hard problems, such as the Steiner tree problem and the travelling salesperson problem, can be solved by using spanning trees and they have wide applications in many areas such as network design, bioinformatics (Biggs, 1993; Brown et al., 1996; Colbourn, 1987; Bermond et al., 1995; Myrvold et al., 1991). The number of spanning trees of $G$, denoted by $\tau(G)$, is the total number of distinct spanning subgraphs of $G$ that are trees. A classic result of Kirchhoff, (Cayley, 1889) can be used to determine the number of spanning trees for $\mathrm{G}=(\mathrm{V}, \mathrm{E})$. Let $\mathrm{V}=\mathrm{v}_{1}, \ldots \ldots \ldots, \mathrm{v}_{\mathrm{n}}$. To state the result, we define the $n \times n$ characteristic matrix $A=\left[a_{i j}\right]$ as follows: (i) $\mathrm{a}_{\mathrm{ij}}=-1$ if $\mathrm{v}_{\mathrm{i}}$ and $\mathrm{v}_{\mathrm{j}}$ are adjacent and $\mathrm{i} \neq \mathrm{j}$, (ii) $\mathrm{a}_{\mathrm{ij}}$ equals the degree of vertex $\mathrm{v}_{\mathrm{i}}$ if $\mathrm{i}=\mathrm{j}$ and (iii) $\mathrm{a}_{\mathrm{ij}}=0$ otherwise. The Kirchhoff matrix tree theorem states that all cofactors of A are equal and their common value is $\tau(\mathrm{G})$. The matrix tree theorem can be applied to any graph $\mathrm{G}$ to determine $\tau(\mathrm{G})$, but this requires evaluating a determinant of a corresponding characteristic matrix. However, for a few special families of graphs there exists simple formulas that make it much easier to calculate and determine the number of corresponding spanning trees especially when these numbers are very large. One of the first such result is due to Cayley who showed that complete graph on $n$ vertices, $K_{n}$ has $\mathrm{n}^{\mathrm{n}-2}$ spanning trees (Cvetkovic et al., 1980) that he showed $\tau\left(K_{n}\right)=n^{n-2}, n \geq 2$. Another result, $\tau\left(K_{n}\right)=p^{q-1}$, $\mathrm{q}^{\mathrm{p}-1} \mathrm{p}, \mathrm{q} \geq 1$, where $\mathrm{K}_{\mathrm{p}, \mathrm{q}}$ is the complete bipartite graph with bipartite sets containing $p$ and $q$ vertices,

Corresponding Author: Daoud, S.N., Department of Mathematics, Faculty of Science, El-Minufiya University, Shebeen El-Kom, Egypt 
respectively. It is well known, as in e.g., (Petingi et al., 1998; Austin, 1960; Clark, 2003; Egecioglu and Remmel, 1994; Porter, 2004; Lewis, 1999).

Let $G_{1}$ and $G_{2}$ be a simple graphs. We introduce some operations on graphs:

- If $\mathrm{G}_{1}$ and $\mathrm{G}_{2}$ are vertex disjoint graphs. Then the join product, $G_{1} \vee G_{2}$, is the super-graph of $G_{1}+G_{2}$, in which each vertex of $G_{1}$ is adjacent to every vertex of $\mathrm{G}_{2}$, (Balakrishnan and Ranganathan, 2000).

- The Cartesian product of two graphs $G_{1}$ and $G_{2}$, $G_{1 \times} G_{2}$, is the simple graph with vertex set $V\left(G_{1 \times}\right.$ $\left.\mathrm{G}_{2}\right)=\mathrm{V}\left(\mathrm{V}_{1} \times \mathrm{V}_{2}\right)$ and edge set $\mathrm{E}\left(\mathrm{G}_{1} \times \mathrm{G}_{2}\right)=$ $\left[\left(\mathrm{E}_{1} \times \mathrm{V}_{2}\right) \cup\left(\mathrm{V}_{1} \times \mathrm{E}_{1}\right)\right]$, such that two vertices $\left(\mathrm{u}_{1}, \mathrm{u}_{2}\right)$ and $\left(\mathrm{v}_{1}, \mathrm{v}_{2}\right)$ are adjacent in $\mathrm{G}_{1} \times \mathrm{G}_{2}$ iff, either $\mathrm{u}_{1}=\mathrm{v}_{1}$ and $\mathrm{u}_{2}$ is adjacent to $\mathrm{v}_{2}$ in $\mathrm{G}_{1}$, or $\mathrm{u}_{1}$ is adjacent to $v_{1}$ in $G_{1}$ and $u_{2}=v_{2}$, (Wilson and Watkins, 1990).

- The tensor product, or Kronecher product of two graphs $G_{1}$ and $G_{2}, G_{1} \otimes G_{2}$, is the simple graph with $\mathrm{v}\left(\mathrm{G}_{1} \otimes \mathrm{G}_{2}\right)=\mathrm{V}_{1} \times \mathrm{V}_{2}$ where $\left(\mathrm{u}_{1}, \mathrm{u}_{2}\right)$ and $\left(\mathrm{v}_{1}\right.$, $v_{2}$ ) are adjacent in $G_{1} \otimes G_{2}$ iff $u_{1}$ is adjacent to $v_{1}$ in $\mathrm{G}_{1}$ and $\mathrm{u}_{2}$ is adjacent to $\mathrm{v}_{2}$ in $\mathrm{G}_{2}$, (Balakrishnan and Ranganathan, 2000).

- The normal product, or the strong produc of two graphs $G_{1}$ and $G_{2} t, G_{1} \circ G_{2}$, is the simple graph with $\mathrm{V}\left(\mathrm{G}_{1} \circ \mathrm{G}_{2}\right)=\mathrm{V}_{1} \times \mathrm{V}_{2}$ where $\left(\mathrm{u}_{1}, \mathrm{u}_{2}\right)$ and $\left(\mathrm{v}_{1}, \mathrm{v}_{2}\right)$ are adjacent in $G_{1} \circ G_{2}$ iff either $u_{1}=v_{1}$ and $u_{2}$ is adjacent to $v_{2}$ in $G_{2}$, or $u_{1}$ is adjacent to $v_{1}$ in $G_{1}$ and $\mathrm{u}_{2}=\mathrm{v}_{2}$, or $\mathrm{u}_{1}$ is adjacent to $\mathrm{v}_{1}$ and $\mathrm{u}_{2}$ is adjacent to $\mathrm{v}_{2}$, (Balakrishnan and Ranganathan, 2000).

- The corona $\mathrm{G}_{1} \odot \mathrm{G}_{2}$ of $\mathrm{G}_{1}$ and $\mathrm{G}_{2}$ is the graph obtained by taking one copy of $G_{1}$ (which has $n_{1}$ vertices) and $n_{1}$ copies of $G_{2}$ and then joining the $i^{\text {th }}$ vertex of $G_{2}$ to every vertex in the $i^{\text {th }}$ copy of $G_{2}$, (Wilson and Watkins, 1990).

The well-known matrix tree theorem (Kirchhoff matrix) can be used to count the number of spanning trees for small graphs, but this method is not feasible for large graphs. So we present two formulas in lemma1, lemma2 is to express $\tau(\mathrm{G})$ directly as a determinant rather than in terms of cofactors as in Kirchhoff theorem.

Lemma 1: Let $\mathrm{G}$ be a simple graph with $\mathrm{n}$ vertices. Then, $\tau(\mathrm{G})=\frac{1}{\mathrm{n}^{2}} \operatorname{det}[\mathrm{D}-\mathrm{A}+\mathrm{U}]$, where $\mathrm{A}, \mathrm{D}$ are the adjacency and degree matrices of $\mathrm{G}$ respectively and $\mathrm{U}$ is the $\mathrm{n} \times \mathrm{n}$ matrix where all its elements are ones.
Proof: By simple calculations using the property of addition of two determinants, we can write $\operatorname{det}[\mathrm{D}-\mathrm{A}+\mathrm{U}]$ as an addition of $\mathrm{n}$ determinants each of which is the same as $\operatorname{det}[\mathrm{D}-\mathrm{A}]$, but by replace one of its column and any determinant of these is equal to $n \times \operatorname{det}[D-A]$. Since all cofactors of [D-A] are equals. Then we have:

$$
\begin{aligned}
& \operatorname{det}[D-A+U]=n \times \operatorname{cofactor}[D-A]+ \\
& n \times \operatorname{cofactor}[D-A]+\ldots \ldots+n \times \text { cofactor } \\
& {[D-A](n-\text { times })+\operatorname{det}[D-A]}
\end{aligned}
$$

But:

$$
\operatorname{det}[\mathrm{D}-\mathrm{A}]=0
$$

Then:

$$
\operatorname{det}[\mathrm{D}-\mathrm{A}+\mathrm{U}]=\mathrm{n}^{2} \times \operatorname{cofactor}[\mathrm{D}-\mathrm{A}]=\mathrm{n}^{2} \tau(\mathrm{G})
$$

Therefore:

$$
\tau(\mathrm{G})=\frac{1}{\mathrm{n}^{2}} \operatorname{det}[\mathrm{D}-\mathrm{A}+\mathrm{U}]
$$

Lemma 2: Let $G$ be a simple graph with vertices $n$. Then, $\tau(G)=\frac{1}{n^{2}} \operatorname{det}\left[\mathrm{nI}_{\mathrm{n}}-\overline{\mathrm{D}}+\overline{\mathrm{A}}\right]$, where $\overline{\mathrm{A}}, \overline{\mathrm{D}}$ are the adjacency and degree matrices of $\overline{\mathrm{G}}$ ( complement of $\mathrm{G})$ respectively and $I_{n}$ is the identity matrix.

Proof: It is clear that $\mathrm{D}+\overline{\mathrm{D}}=(\mathrm{n}-1) \mathrm{I}_{\mathrm{n}} \quad$ and $\left(\mathrm{U}-\mathrm{A}-\mathrm{I}_{\mathrm{n}}\right)=\overline{\mathrm{A}}$.Then.

$\mathrm{D}-\mathrm{A}+\mathrm{U}=(\mathrm{n}-1) \mathrm{I}_{\mathrm{n}}-\overline{\mathrm{D}}+\overline{\mathrm{A}}+\mathrm{I}_{\mathrm{n}}=n I_{\mathrm{n}}-\overline{\mathrm{D}}+\overline{\mathrm{A}}$. Thus from lemma1, we have: $\tau(G)=\frac{1}{n^{2}} \operatorname{det}\left[\mathrm{nI}_{\mathrm{n}}-\overline{\mathrm{D}}+\overline{\mathrm{A}}\right]$.

Theorem 3: $\tau\left(K_{n}\right)=n^{n-2}$.

Proof: Applying lemma 2, we have:

$$
\begin{aligned}
& \tau\left(\mathrm{K}_{\mathrm{n}}\right)=\frac{1}{\mathrm{n}^{2}} \operatorname{det}(\mathrm{nI}-\overline{\mathrm{D}}+\overline{\mathrm{A}})= \\
& \frac{1}{\mathrm{n}^{2}} \operatorname{det}\left(\begin{array}{ccccc}
\mathrm{n} & 0 & 0 & \cdots & 0 \\
0 & \mathrm{n} & 0 & \ddots & \vdots \\
0 & \ddots & \ddots & \ddots & 0 \\
\vdots & \ddots & \ddots & \mathrm{n} & 0 \\
0 & \cdots & 0 & 0 & \mathrm{n}
\end{array}\right)=\frac{1}{\mathrm{n}^{2}} \times \mathrm{n}^{\mathrm{n}}=\mathrm{n}^{\mathrm{n}-2}
\end{aligned}
$$

Corollary 4: $\tau\left(K_{n}-e\right)=(n-2) n^{n-3}$. 
Proof: Applying lemma 2, we have:

$$
\begin{aligned}
\tau\left(\mathrm{K}_{\mathrm{n}}-\mathrm{e}\right) & =\frac{1}{\mathrm{n}^{2}} \operatorname{det}(\mathrm{n} I-\overline{\mathrm{D}}+\overline{\mathrm{A}}) \\
& =\frac{1}{\mathrm{n}^{2}} \operatorname{det}\left(\begin{array}{ccccc}
\mathrm{n}-1 & 1 & 0 & \cdots & 0 \\
1 & \mathrm{n}-1 & 0 & \ddots & \vdots \\
0 & \ddots & \ddots & \ddots & 0 \\
\vdots & \ddots & \ddots & \mathrm{n} & 0 \\
0 & \cdots & 0 & 0 & \mathrm{n}
\end{array}\right) \\
& =\frac{1}{\mathrm{n}^{2}} \times\left((\mathrm{n}-1)^{2}-1\right) \times \mathrm{n}^{\mathrm{n}-2}=(\mathrm{n}-2) \times \mathrm{n}^{\mathrm{n}-3}
\end{aligned}
$$

Corollary 5: $\tau\left(K_{n} \circ e\right)=2 n^{n-3}$, where $K_{n} \circ e$ is the graph obtained from $K_{n}$ by contracting the edge e .

Proof: Immediately from the fact that:

$$
\tau(\mathrm{G})=\tau(\mathrm{G}-\mathrm{e})+\tau(\mathrm{G} \circ \mathrm{e})
$$

Theorem 6: Let $\mathrm{G}$ be a graph constructed by removing $m$ distinct edges from $K_{n}, n \geq 2 m$. Then:

$$
\tau(\mathrm{G})=\mathrm{n}^{\mathrm{n}-2}\left(1-\frac{2}{\mathrm{n}}\right)^{\mathrm{m}}
$$

Proof: Straightforward induction using properties of determinants.

Theorem 7: $\tau\left(K_{n}+e\right)=(n+2) n^{n-3}$.

Proof: Applying lemma 2, we have:

$$
\begin{aligned}
& \tau\left(\mathrm{K}_{\mathrm{n}}+\mathrm{e}\right)=\frac{1}{\mathrm{n}^{2}} \operatorname{det}(\mathrm{n} I-\overline{\mathrm{D}}+\overline{\mathrm{A}})= \\
& \frac{1}{\mathrm{n}^{2}} \operatorname{det}\left(\begin{array}{ccccc}
\mathrm{n}+1 & -1 & 0 & \cdots & 0 \\
-1 & \mathrm{n}+1 & 0 & \ddots & \vdots \\
0 & \ddots & \mathrm{n} & \ddots & 0 \\
\vdots & \ddots & \ddots & \ddots & 0 \\
0 & \cdots & 0 & 0 & \mathrm{n}
\end{array}\right) \\
& \quad=\frac{1}{\mathrm{n}^{2}} \times\left((\mathrm{n}+1)^{2}-1\right) \times \mathrm{n}^{\mathrm{n}-2}=(\mathrm{n}+2) \times \mathrm{n}^{\mathrm{n}-3}
\end{aligned}
$$

Theorem 8: Let $\mathrm{H}_{\mathrm{n}}$ be a graph constructed by removing $\mathrm{n}$ distinct edges from $\mathrm{K}_{2 \mathrm{n}}$. Then:

$$
\tau\left(\mathrm{H}_{\mathrm{n}}\right)=2^{2 \mathrm{n}-2} \times \mathrm{n}^{\mathrm{n}-1} \times(\mathrm{n}-1)^{\mathrm{n}}
$$

Proof: Applying lemma 2, we have:

$$
\tau\left(H_{n}\right)=\frac{1}{(2 n)^{2}} \operatorname{det}(2 n I-\bar{D}+\bar{A})=\frac{1}{(2 n)^{2}} \operatorname{det}
$$

$$
\left(\begin{array}{cccccccccc}
2 \mathrm{n}-1 & 1 & 0 & \cdots & \cdots & \cdots & \cdots & \cdots & \cdots & 0 \\
1 & 2 \mathrm{n}-1 & 0 & \ddots & \vdots & \ddots & \ddots & \ddots & & \vdots \\
0 & 0 & 2 \mathrm{n}-1 & 1 & 0 & \vdots & \ddots & \ddots & \ddots & \vdots \\
\vdots & \ddots & 1 & 2 \mathrm{n}-1 & 0 & \ddots & & & \ddots & \vdots \\
\vdots & \cdots & 0 & 0 & 2 \mathrm{n}-1 & 1 & 0 & \cdots & \cdots & \vdots \\
\vdots & \vdots & & \ddots & 1 & 2 \mathrm{n}-1 & 0 & \cdots & \cdots & \vdots \\
\vdots & \vdots & \ddots & & \ddots & 0 & \ddots & 0 & \ddots & \vdots \\
& \ddots & \ddots & \ddots & \vdots & 0 & 1 & \ddots & & 0 \\
& & \ddots & \ddots & \ddots & \vdots & \ddots & \ddots & 2 \mathrm{n}-1 & 1 \\
0 & \cdots & \cdots & \cdots & \cdots & \cdots & \cdots & 0 & 1 & 2 \mathrm{n}-1
\end{array}\right)
$$

Straightforward induction using properties of determinants. We have:

$$
\tau\left(\mathrm{H}_{\mathrm{n}}\right)=\frac{1}{(2 \mathrm{n})^{2}} \times\left((2 \mathrm{n}-1)^{2}-1\right)^{\mathrm{n}}=2^{2 \mathrm{n}-2} \times \mathrm{n}^{\mathrm{n}-1} \times(\mathrm{n}-1)^{\mathrm{n}}
$$

Theorem 9: Let $G$ be a graph constructed by removing a star graph $\mathrm{K}_{1,2}$ from $\mathrm{K}_{\mathrm{n}}$. Then:

$$
\tau(\mathrm{G})=\mathrm{n}^{\mathrm{n}-2}\left(1-\frac{1}{\mathrm{n}}\right)\left(1-\frac{3}{\mathrm{n}}\right)
$$

Proof: Apply lemma 2, we have:

$$
\begin{gathered}
\tau(\mathrm{G})=\frac{1}{\mathrm{n}^{2}} \operatorname{det}(\mathrm{n} I-\overline{\mathrm{D}}+\overline{\mathrm{A}})=\frac{1}{\mathrm{n}^{2}} \operatorname{det} \\
\left(\begin{array}{ccccccc}
\mathrm{n}-2 & 1 & 1 & 0 & \cdots & \cdots & \cdots \\
1 & \mathrm{n}-1 & 0 & \ddots & \vdots & \ddots & \ddots \\
1 & 0 & \mathrm{n}-1 & 0 & 0 & \vdots & \ddots \\
0 & \ddots & 0 & \mathrm{n} & 0 & \ddots & \\
\vdots & \cdots & 0 & 0 & \ddots & 0 & 0 \\
\vdots & \vdots & & \ddots & 0 & \ddots & 0 \\
\vdots & \vdots & \ddots & & \ddots & 0 & \mathrm{n}
\end{array}\right) \\
=\frac{1}{\mathrm{n}^{2}} \operatorname{det}\left(\begin{array}{ccc}
\mathrm{n}-2 & 1 & 1 \\
1 & \mathrm{n}-1 & 0 \\
1 & 0 & \mathrm{n}-1
\end{array}\right) \times \operatorname{det}\left(\begin{array}{ccc}
\mathrm{n} & 0 & \cdots \\
0 & \mathrm{n} & \ddots \\
\vdots & \ddots & \mathrm{n}
\end{array}\right) \\
=\frac{1}{\mathrm{n}^{2}} \times \mathrm{n}^{\mathrm{n}-3} \times(\mathrm{n}-1)\left(\mathrm{n}^{2}-3 \mathrm{n}\right)=\mathrm{n}^{\mathrm{n}-2} \times\left(1-\frac{1}{\mathrm{n}}\right) \times\left(1-\frac{3}{\mathrm{n}}\right)
\end{gathered}
$$

Corollary 10: Let $G$ be a graph constructed by removing a star graph $\mathrm{K}_{1, \mathrm{~m}}$ from $\mathrm{Kn}, \mathrm{n} \geq \mathrm{m}+1$.. Then:

$$
\tau(\mathrm{G})=\mathrm{n}^{\mathrm{n}-2}\left(1-\frac{1}{\mathrm{n}}\right)^{\mathrm{m}-1}\left(1-\frac{\mathrm{m}+1}{\mathrm{n}}\right)
$$

Proof: Straightforward induction using properties of determinants.

Lemma 11: Biggs (1993) let $G$ be a k-regular graph with $n$ vertices and $m$ edges. Then: 


$$
\tau(\mathrm{L}(\mathrm{G}))=2^{\mathrm{m}-\mathrm{n}+1} \times \mathrm{k}^{\mathrm{m}-\mathrm{n}-1} \times \tau(\mathrm{G})
$$

where, $L(G)$ is the line graph of $G$.

Theorem 12: Let $T_{n}$ be the line graph of $K_{n}$. Then:

$$
\begin{aligned}
& \tau\left(T_{n}\right)=\tau\left(L\left(K_{n}\right)\right)= \\
& 2^{\frac{1}{2}\left(n^{2}-3 n+2\right)} \times(n-1)^{\frac{1}{2}\left(n^{2}-3 n-2\right)} \times n^{n-2}
\end{aligned}
$$

Proof: The line graph $\mathrm{L}(\mathrm{G})$ of a graph $\mathrm{G}$ is constructed by taking the edges of $G$ as vertices of $\mathrm{L}(\mathrm{G})$ and joining two vertices in $\mathrm{L}(\mathrm{G})$ whenever the corresponding edges in $\mathrm{G}$ have a common vertex. Also if $G$ is regular of valency $k$, its line graph $L(G)$ is regular of valency $2 \mathrm{k}-2$. It is easy to show that $\mathrm{L}\left(\mathrm{K}_{\mathrm{n}}\right)$ is the triangle graph $\mathrm{T}_{\mathrm{n}}$ which can be described by saying that the $\frac{1}{2} n(n-1)$ pairs of numbers from the set $\{1,2, \ldots, n\}$, two vertices being adjacent whenever the corresponding pairs have just one common member. Appling lemma11 taking $\mathrm{k}=\mathrm{n}-1, \mathrm{~m}=\frac{1}{2} \mathrm{n}(\mathrm{n}-1)$, we have:

$$
\tau\left(T_{n}\right)=\tau\left(L\left(K_{n}\right)\right)=2^{\frac{1}{2}\left(n^{2}-3 n+2\right)} \times(n-1)^{\frac{1}{2}\left(n^{2}-3 n-2\right)} \times n^{n-2}
$$

Lemma 13: Let $A_{n}(x)$ be $n \times n$ matrix such that:

$$
\mathrm{A}_{\mathrm{n}}(\mathrm{x})=\left(\begin{array}{cccccc}
\mathrm{x} & 1 & 1 & \cdots & \cdots & 1 \\
1 & \mathrm{x} & 1 & \ddots & & \vdots \\
1 & \ddots & \ddots & 1 & \ddots & \vdots \\
\vdots & \ddots & \ddots & \ddots & \ddots & 1 \\
\vdots & & \ddots & \ddots & \mathrm{x} & 1 \\
1 & \cdots & \cdots & 1 & 1 & \mathrm{x}
\end{array}\right)
$$

Then:

$$
\operatorname{det}\left(A_{n}\right)=(x+n-1)(x-1)^{n-1}
$$

Proof: From the definition of the circulant determinants, we have:

$$
\begin{aligned}
\operatorname{det}\left(\mathrm{A}_{\mathrm{n}}(\mathrm{x})\right)= & \prod_{\mathrm{j}=1}^{\mathrm{n}}\left(\mathrm{x}+\omega_{\mathrm{j}}+\omega_{\mathrm{j}}^{2}+\omega_{\mathrm{j}}^{3}+\ldots \ldots \ldots . . . \omega_{\mathrm{j}}^{\mathrm{n}-1}\right) \\
= & (\mathrm{x}+1+1+\ldots \ldots+1) \times \prod_{\mathrm{j}=1, \omega_{\mathrm{j}} \neq 1}^{\mathrm{n}} \\
& (\mathrm{x}+\underbrace{\omega_{\mathrm{j}}+\omega_{\mathrm{j}}^{2}+\omega_{\mathrm{j}}^{3}+\ldots \ldots \ldots . . \omega_{\mathrm{j}}^{\mathrm{n}-1}}_{=-1}) \\
= & (\mathrm{x}+\mathrm{n}-1) \times(\mathrm{x}-1)^{\mathrm{n}-1} .
\end{aligned}
$$

Lemma14: If $H=\left(\begin{array}{cc}A & B \\ B & A\end{array}\right)$ and $A B=B A$. Then $\operatorname{det}(H)=\operatorname{det}(A+B) \cdot \operatorname{det}(A-B)$.

Proof: Using the fact that $\operatorname{det}\left(\begin{array}{ll}A & B \\ C & D\end{array}\right)=\left\{\begin{array}{l}\operatorname{det}(A) \cdot \operatorname{det}\left(D-C A^{-1} B\right), \text { where A,B } \\ \operatorname{det}(D) \cdot \operatorname{det}\left(A-B D^{-1} C\right)\end{array}\right.$ are non singular, Marcus $\mathrm{M}$. [12]. We have $\begin{aligned} \operatorname{det}\left(\begin{array}{ll}A & B \\ B & A\end{array}\right) & =\operatorname{det}(A) \cdot \operatorname{det}\left(A-B A^{-1} B\right) \\ & =\operatorname{det}\left(A^{2}-B^{2}\right)=\operatorname{det}(A+B) \cdot \operatorname{det}(A-B) \cdot\end{aligned}$

This formula gives some sort of symmetry in some matrices which facilitates our calculation of determinants.

Theorem 15: $\tau\left(K_{2} \times K_{n}\right)=n^{n-2} \times(n+2)^{n-1}$.

Proof: Applying lemmas 2. We have:

$\tau\left(K_{2} \times K_{n}\right)=\frac{1}{(2 n)^{2}} \operatorname{det}(2 n I-\bar{D}+\bar{A})=\frac{1}{(2 n)^{2}} \operatorname{det}$

$\left(\begin{array}{cccccccccc}\mathrm{n}+1 & 0 & \cdots & \cdots & 0 & 0 & 1 & \cdots & \cdots & 1 \\ 0 & \mathrm{n}+1 & 0 & \ddots & \vdots & 1 & 0 & 1 & \cdots & \vdots \\ \vdots & 0 & \ddots & \ddots & \vdots & \vdots & \ddots & \ddots & \ddots & \vdots \\ \vdots & \ddots & \ddots & \mathrm{n}+1 & 0 & \vdots & & 1 & 0 & 1 \\ 0 & \cdots & \ldots & 0 & \mathrm{n}+1 & 1 & \cdots & \ldots & 1 & 0 \\ 0 & 1 & \cdots & \cdots & 1 & \mathrm{n}+1 & 0 & \ldots & \cdots & 0 \\ 1 & 0 & 1 & \ldots & \vdots & 0 & \mathrm{n}+1 & 0 & \ddots & \vdots \\ & \ddots & \ddots & \ddots & \vdots & \vdots & 0 & \ddots & & \vdots \\ & & \ddots & 0 & 1 & \vdots & \ddots & \ddots & \mathrm{n}+1 & 0 \\ 1 & \cdots & \cdots & 1 & 0 & 0 & \cdots & \cdots & 0 & \mathrm{n}+1\end{array}\right)$

Using lemma14, we get:

$\tau\left(\mathrm{K}_{2} \times \mathrm{K}_{\mathrm{n}}\right)=$

$\frac{1}{(2 \mathrm{n})^{2}} \operatorname{det}\left(\begin{array}{ccccc}\mathrm{n}+1 & 1 & 1 & \cdots & 1 \\ 1 & \mathrm{n}+1 & 1 & \ddots & \vdots \\ 1 & 1 & \ddots & \ddots & 1 \\ \vdots & \ddots & \ddots & \mathrm{n}+1 & 1 \\ 1 & \cdots & 1 & 1 & \mathrm{n}+1\end{array}\right) \times \operatorname{det}\left(\begin{array}{ccccc}\mathrm{n}+1 & -1 & \cdots & \cdots & -1 \\ -1 & \mathrm{n}+1 & -1 & \ddots & \vdots \\ \vdots & \ddots & \ddots & \ddots & \vdots \\ \vdots & \ddots & \ddots & \mathrm{n}+1 & -1 \\ -1 & \cdots & \cdots & -1 & \mathrm{n}+1\end{array}\right)$

Applying lemma13 with $\mathrm{x}=\mathrm{n}+1$, for the first determinant and properties of determinants for the second, yields:

$$
\tau\left(\mathrm{K}_{2} \times \mathrm{K}_{\mathrm{n}}\right)=\frac{1}{(2 \mathrm{n})^{2}} \times 2 \mathrm{n}^{\mathrm{n}} \times 2(\mathrm{n}+2)^{\mathrm{n}-1}=(\mathrm{n}+2)^{\mathrm{n}-1} \times \mathrm{n}^{\mathrm{n}-2}
$$

Theorem 16: $\tau\left(K_{2} \otimes K_{n}\right)=n^{n-2}(n-1)(n-2)^{n-1}$.

Proof: Applying lemmas 2. We have: 
Am. J. Applied Sci., 9 (4): 584-592, 2012

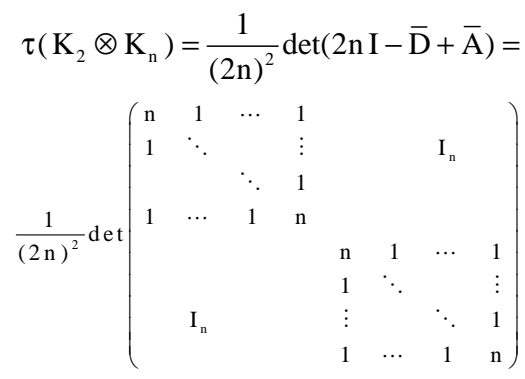

Using lemma14, we get:

$$
\begin{gathered}
\tau\left(\mathrm{K}_{2} \otimes \mathrm{K}_{\mathrm{n}}\right)= \\
\frac{1}{(2 \mathrm{n})^{2}} \operatorname{det}\left(\begin{array}{ccccc}
\mathrm{n}+1 & 1 & 1 & \ldots & 1 \\
1 & \mathrm{n}+1 & 1 & \ddots & \vdots \\
1 & 1 & \ddots & \ddots & 1 \\
\vdots & \ddots & \ddots & \mathrm{n}+1 & 1 \\
1 & \cdots & 1 & 1 & \mathrm{n}+1
\end{array}\right) \times \operatorname{det}\left(\begin{array}{ccccc}
\mathrm{n}-1 & 1 & 1 & \ldots & 1 \\
1 & \mathrm{n}-1 & 1 & \ddots & \vdots \\
1 & 1 & \ddots & \ddots & 1 \\
\vdots & \ddots & \ddots & \mathrm{n}-1 & 1 \\
1 & \cdots & 1 & 1 & \mathrm{n}-1
\end{array}\right)
\end{gathered}
$$

Applying lemma13 with $\mathrm{x}=\mathrm{n}+1, \mathrm{n}-1$ respectively, we have:

$$
\begin{aligned}
\tau\left(K_{2} \otimes K_{n}\right) & =\frac{1}{(2 n)^{2}} \times 2 n^{n} \times 2(n-1)(n-2)^{n-1} \\
& =n^{n-2}(n-1)(n-2)^{n-1}
\end{aligned}
$$

Theorem 17: $\tau\left(K_{2} \circ K_{n}\right)=(2 n)^{2 n-2}$.

Proof: Applying lemma 2, we have:

$$
\begin{gathered}
\tau\left(\mathrm{K}_{2} \circ \mathrm{K}_{\mathrm{n}}\right)=\frac{1}{(2 \mathrm{n})^{2}} \operatorname{det}(2 \mathrm{nI}-\overline{\mathrm{D}}+\overline{\mathrm{A}})=\frac{1}{(2 \mathrm{n})^{2}} \operatorname{det} \\
\left(\begin{array}{cccccccccc}
2 \mathrm{n} & 0 & \cdots & \cdots & 0 & 0 & \cdots & \cdots & \cdots & 0 \\
0 & 2 \mathrm{n} & 0 & \ddots & \vdots & \vdots & 0 & \cdots & \cdots & \vdots \\
\vdots & 0 & \ddots & \ddots & \vdots & \vdots & \ddots & \ddots & \ddots & \vdots \\
\vdots & \ddots & \ddots & 2 \mathrm{n} & 0 & \vdots & \cdots & \ddots & \ddots & \vdots \\
0 & \cdots & \cdots & 0 & 2 \mathrm{n} & 0 & \cdots & \cdots & \cdots & 0 \\
0 & \cdots & \cdots & \cdots & 0 & 2 \mathrm{n} & 0 & \cdots & \cdots & 0 \\
\vdots & 0 & \cdots & \cdots & \vdots & 0 & 2 \mathrm{n} & 0 & \ddots & \vdots \\
& \ddots & \ddots & \ddots & \vdots & \vdots & 0 & \ddots & & \vdots \\
0 & \cdots & \cdots & \cdots & 0 & 0 & \cdots & \cdots & 0 & 2 \mathrm{n}
\end{array}\right) \\
=\frac{1}{(2 \mathrm{n})^{2}}(2 \mathrm{n})^{\mathrm{n}} \times(2 \mathrm{n})^{\mathrm{n}}=(2 \mathrm{n})^{2 \mathrm{n}-2}=\tau\left(\mathrm{K}_{2 \mathrm{n}}\right)
\end{gathered}
$$

Theorem 18: $\tau\left(K_{2} \odot K_{n}\right)=n^{2(n-2)}$.

Proof: Applying lemma 2, we have:

$$
\tau\left(K_{2} \odot K_{n}\right)=\frac{1}{(2 n)^{2}} \operatorname{det}(2 n I-\bar{D}+\bar{A})=\frac{1}{(2 n)^{2}} \operatorname{det}
$$

$$
\left(\begin{array}{cccccccccc}
\mathrm{n}+1 & 0 & \cdots & \cdots & 0 & 0 & 1 & \cdots & \cdots & 1 \\
0 & \mathrm{n} & 0 & \ddots & \vdots & 1 & 1 & \cdots & \cdots & \vdots \\
\vdots & 0 & \ddots & \ddots & \vdots & \vdots & \ddots & \ddots & & \vdots \\
\vdots & \ddots & \ddots & \mathrm{n} & 0 & \vdots & \cdots & \ddots & \ddots & \vdots \\
0 & \cdots & \cdots & 0 & \mathrm{n} & 1 & \cdots & \cdots & \cdots & 1 \\
0 & 1 & \cdots & \cdots & 1 & \mathrm{n}+1 & 0 & \cdots & \cdots & 0 \\
1 & 1 & \cdots & \cdots & \vdots & 0 & \mathrm{n} & 0 & \ddots & \vdots \\
\vdots & \ddots & \ddots & \ddots & \vdots & \vdots & 0 & \ddots & & \vdots \\
\vdots & & \ddots & \cdots & \vdots & \vdots & \ddots & \ddots & \mathrm{n} & 0 \\
1 & \cdots & \cdots & \cdots & 1 & 0 & \cdots & \cdots & 0 & \mathrm{n}
\end{array}\right)
$$

Using lemma14, we get:

$$
\begin{aligned}
& \tau\left(\mathrm{K}_{2} \odot \mathrm{K}_{\mathrm{n}}\right)= \\
& \frac{1}{(2 \mathrm{n})^{2}} \operatorname{det}\left(\begin{array}{cccc}
\mathrm{n}+1 & 1 & 1 & 1 \\
1 & \mathrm{n}+1 & 1 & \vdots \\
1 & 1 & \ddots & \vdots \\
\vdots & \ldots & \ddots & \mathrm{n}+1
\end{array}\right) \times \operatorname{det}\left(\begin{array}{cccc}
\mathrm{n}+1 & -1 & \cdots & -1 \\
-1 & \mathrm{n}-1 & -1 & \ddots \\
\vdots & \ddots & \ddots & -1 \\
-1 & \cdots & -1 & \mathrm{n}-1
\end{array}\right)
\end{aligned}
$$

Applying lemma13 with $\mathrm{x}=\mathrm{n}+1$ for the first determinant and properties of determinants for the second, yields:

$$
\tau\left(\mathrm{K}_{2} \odot \mathrm{K}_{\mathrm{n}}\right)=\frac{1}{(2 \mathrm{n})^{2}} \times 2 \mathrm{n}^{\mathrm{n}} \times 2 \mathrm{n}^{\mathrm{n}-2}=\mathrm{n}^{2(\mathrm{n}-2)}
$$

Theorem 19: $\tau\left(\mathrm{K}_{\mathrm{n}, \mathrm{m}}\right)=\mathrm{n}^{\mathrm{m}-1} \mathrm{~m}^{\mathrm{n}-1}$.

Proof: Apply lemma 2, we have:

$$
\begin{gathered}
\tau\left(\mathrm{K}_{\mathrm{n}, \mathrm{m}}\right)=\frac{1}{(\mathrm{n}+\mathrm{m})^{2}} \operatorname{det}((\mathrm{n}+\mathrm{m}) \mathrm{I}-\overline{\mathrm{D}}+\overline{\mathrm{A}})=\frac{1}{(\mathrm{n}+\mathrm{m})^{2}} \operatorname{det} \\
\left(\begin{array}{cccccccc}
\mathrm{m}+1 & 1 & \ldots & 1 & 0 & \cdots & \cdots & 0 \\
\vdots & \ddots & \vdots & \vdots & \vdots & \cdots & \cdots & \vdots \\
\vdots & \ddots & \ddots & 1 & \vdots & \cdots & \cdots & \vdots \\
1 & \ddots & 1 & \mathrm{~m}+1 & 0 & \cdots & \cdots & 0 \\
0 & \ldots & \ldots & 0 & \mathrm{n}+1 & 1 & \cdots & 1 \\
\vdots & \cdots & \ldots & \vdots & 1 & \ddots & \vdots & \vdots \\
\vdots & \cdots & \ldots & \vdots & \vdots & \ddots & \ddots & 1 \\
0 & \ldots & \ldots & 0 & 1 & \cdots & 1 & \mathrm{n}+1
\end{array}\right)
\end{gathered}
$$

Using lemma14, we get:

$$
\begin{aligned}
& \tau\left(\mathrm{K}_{\mathrm{n}, \mathrm{m}}\right)= \\
& \frac{1}{(\mathrm{n}+\mathrm{m})^{2}} \operatorname{det}\left(\begin{array}{cccc}
\mathrm{m}+1 & 1 & \cdots & 1 \\
1 & \ddots & & \vdots \\
\vdots & & \ddots & 1 \\
1 & \cdots & 1 & \mathrm{~m}+1
\end{array}\right) \times \operatorname{det}\left(\begin{array}{cccc}
\mathrm{n}+1 & 1 & \cdots & 1 \\
1 & \ddots & & \vdots \\
\vdots & & \ddots & 1 \\
1 & \cdots & 1 & \mathrm{n}+1
\end{array}\right)
\end{aligned}
$$


Am. J. Applied Sci., 9 (4): 584-592, 2012

Applying lemma13 with $\mathrm{x}=\mathrm{n}+1$ and $\mathrm{x}=\mathrm{m}+1$ respectively, we have:

$$
\begin{aligned}
& \tau\left(K_{n, m}\right)=\frac{1}{(n+m)^{2}} . \\
& (n+m) \times m^{n-1} \times(n+m) \times n^{m-1}=m^{n-1} n^{m-1}
\end{aligned}
$$

Specially, $\tau\left(K_{n, n}\right)=n^{2 n-2}$.

Corollary 20: $\tau\left(K_{n, n}-e\right)=n^{2 n-4} \times(n-1)^{2}$.

Proof: Applying lemma 2, we have:

$$
\begin{gathered}
\tau\left(\mathrm{K}_{\mathrm{n}, \mathrm{n}}-\mathrm{e}\right)=\frac{1}{(2 \mathrm{n})^{2}} \operatorname{det}(2 \mathrm{nI}-\overline{\mathrm{D}}+\overline{\mathrm{A}})=\frac{1}{(2 \mathrm{n})^{2}} \operatorname{det} \\
\left(\begin{array}{cccccccc}
\mathrm{n} & 1 & \ldots & 1 & 1 & 0 & \cdots & 0 \\
\vdots & \mathrm{n}+1 & \vdots & \vdots & 0 & 0 & \cdots & \vdots \\
\vdots & \ddots & \ddots & 1 & \vdots & \cdots & \cdots & \vdots \\
1 & \ddots & 1 & \mathrm{n}+1 & 0 & \cdots & \cdots & 0 \\
1 & 0 & \cdots & 0 & \mathrm{n} & 1 & \cdots & 1 \\
0 & 0 & \cdots & \vdots & 1 & \mathrm{n}+1 & \vdots & \vdots \\
\vdots & \cdots & \cdots & \vdots & \vdots & \ddots & \ddots & 1 \\
0 & \cdots & \cdots & 0 & 1 & \cdots & 1 & \mathrm{n}+1
\end{array}\right)
\end{gathered}
$$

Using lemma14, we get:

$$
\begin{aligned}
& \tau\left(\mathrm{K}_{\mathrm{n}, \mathrm{n}}-\mathrm{e}\right)= \\
& \frac{1}{(2 \mathrm{n})^{2}} \operatorname{det}\left(\begin{array}{cccc}
\mathrm{n}+1 & 1 & \cdots & 1 \\
1 & \ddots & & \vdots \\
\vdots & & \ddots & 1 \\
1 & \cdots & 1 & \mathrm{n}+1
\end{array}\right) \times \operatorname{det}\left(\begin{array}{cccc}
\mathrm{n}-1 & 1 & \cdots & 1 \\
1 & \mathrm{n}+1 & & \vdots \\
\vdots & & \ddots & 1 \\
1 & \cdots & 1 & \mathrm{n}+1
\end{array}\right)
\end{aligned}
$$

Applying lemma13 with $\mathrm{x}=\mathrm{n}+1$ for the first determinant and properties of determinants for the second, yields:

$\tau\left(K_{n, n}-e\right)=\frac{1}{(2 n)^{2}} \times 2 n^{n} \times 2 n^{n-2} \times(n-1)^{2}=n^{2 n-4} \times(n-1)^{2}$

Theorem 21: $\tau\left(K_{n, n}+e\right)=n^{2(n-2)}\left(n^{2}+2 n-1\right)$.

Proof: Applying lemma 2, we have:

$$
\tau\left(K_{n, n}+e\right)=\frac{1}{(2 n)^{2}} \operatorname{det}(2 n I-\bar{D}+\bar{A})=\frac{1}{(2 n)^{2}} \operatorname{det}
$$

$$
\left(\begin{array}{cccccccccc}
\mathrm{n}+2 & 1 & \cdots & \cdots & 1 & -1 & 0 & \cdots & \cdots & 0 \\
1 & \mathrm{n}+1 & 1 & \ddots & \vdots & 0 & 0 & \cdots & \cdots & \vdots \\
\vdots & 1 & \ddots & \ddots & \vdots & \vdots & \ddots & \ddots & \ddots & \vdots \\
\vdots & \ddots & \ddots & \mathrm{n}+1 & 1 & \vdots & \cdots & \ddots & \ddots & \vdots \\
1 & \cdots & \cdots & 1 & \mathrm{n}+1 & 0 & \cdots & \cdots & \cdots & 0 \\
-1 & 0 & \cdots & \cdots & 0 & \mathrm{n}+2 & 1 & \cdots & \cdots & 1 \\
0 & 0 & \cdots & \cdots & \vdots & 1 & \mathrm{n}+1 & 1 & \ddots & \vdots \\
\vdots & \ddots & \ddots & \ddots & \vdots & \vdots & 1 & \ddots & & \vdots \\
\vdots & \ddots & \ddots & \cdots & \vdots & \vdots & \ddots & \ddots & \mathrm{n}+1 & 1 \\
0 & \cdots & \cdots & \cdots & 0 & 1 & \cdots & \cdots & 1 & \mathrm{n}+1
\end{array}\right)
$$

Using lemma14, we get:

$$
\begin{gathered}
\tau\left(\mathrm{K}_{\mathrm{n}, \mathrm{n}}+\mathrm{e}\right)= \\
\frac{1}{(2 \mathrm{n})^{2}} \operatorname{det}\left(\begin{array}{ccccc}
\mathrm{n}+1 & 1 & 1 & \cdots & 1 \\
1 & \mathrm{n}+1 & 1 & \ddots & \vdots \\
1 & 1 & \ddots & \ddots & 1 \\
\vdots & \ddots & \ddots & \mathrm{n}+1 & 1 \\
1 & \cdots & 1 & 1 & \mathrm{n}+1
\end{array}\right) \times \operatorname{det}\left(\begin{array}{ccccc}
\mathrm{n}+3 & 1 & 1 & \cdots & 1 \\
1 & \mathrm{n}+1 & 1 & \ddots & \vdots \\
1 & 1 & \ddots & \ddots & 1 \\
\vdots & \ddots & \ddots & \mathrm{n}+1 & 1 \\
1 & \cdots & 1 & 1 & \mathrm{n}+1
\end{array}\right)
\end{gathered}
$$

Applying lemma13 with $\mathrm{x}=\mathrm{n}+1$ for the first determinant and properties of determinants for the for the second, yields:

$$
\begin{aligned}
\tau\left(K_{n, n}+e\right) & =\frac{1}{(2 n)^{2}} \times 2 n^{n} \times 2 n^{n-2}\left(n^{2}+2 n-1\right) \\
& =n^{2(n-2)}\left(n^{2}+2 n-1\right)
\end{aligned}
$$

Theorem 22: $\tau\left(L\left(K_{n, n}\right)\right)=2^{n^{2}-2 n+1} \times n^{n^{2}-2 n-1} \times n^{2(n-1)}$.

Proof: It is easy to show that $L\left(K_{n, n}\right)$ is the $K_{n} \times K_{n}$. Appling lemma11 taking $\mathrm{k}=\mathrm{n}$ and $\mathrm{m}=\mathrm{n}^{2}$. We have:

$\tau\left(L\left(K_{n, n}\right)\right)=\tau\left(K_{n} \times K_{n}\right)=2^{n^{2}-2 n+1} \times n^{n^{2}-2 n-1} \times n^{2(n-1)}$

Theorem23:

$\tau\left(\mathrm{K}_{1}+\mathrm{K}_{\mathrm{n}, \mathrm{m}}\right)=(\mathrm{n}+\mathrm{m}+1) \times(\mathrm{n}+1)^{\mathrm{m}-1} \times(\mathrm{m}+1)^{\mathrm{n}-1}$.

Proof: Applying lemma 2, we have:

$$
\begin{aligned}
& \tau\left(K_{1}+K_{n, m}\right)=\frac{1}{(n+m+1)^{2}} \operatorname{det}[(n+m+1) I-\bar{D}+\bar{A}] \\
& =(\mathrm{n}+\mathrm{m}+1) \times \frac{1}{(\mathrm{n}+\mathrm{m}+1)^{2}} \mathrm{det} \\
& \left(\begin{array}{ccccccccc}
\mathrm{m}+2 & 1 & \cdots & 1 & 0 & \cdots & \cdots & 0 & 0 \\
\vdots & \ddots & \vdots & \vdots & \vdots & \cdots & \cdots & \vdots & \vdots \\
\vdots & \ddots & \ddots & 1 & \vdots & \cdots & \cdots & \vdots & \vdots \\
1 & \ddots & 1 & \mathrm{~m}+2 & 0 & \cdots & \cdots & 0 & \vdots \\
0 & \cdots & \cdots & 0 & \mathrm{n}+2 & 1 & \cdots & 1 & \vdots \\
\vdots & \cdots & \cdots & \vdots & 1 & \ddots & \vdots & \vdots & \vdots \\
\vdots & \cdots & \cdots & \vdots & \vdots & \ddots & \ddots & 1 & \vdots \\
0 & \cdots & \cdots & 0 & 1 & \cdots & 1 & \mathrm{n}+2 & 0 \\
0 & \cdots & \cdots & \cdots & \cdots & \cdots & \cdots & \cdots & \mathrm{n}+\mathrm{m}+1
\end{array}\right)
\end{aligned}
$$




$$
\begin{aligned}
& =(\mathrm{n}+\mathrm{m}+1) \times \frac{1}{(\mathrm{n}+\mathrm{m}+1)^{2}} \operatorname{det} \\
& \left(\begin{array}{cccc}
\mathrm{m}+2 & 1 & \cdots & 1 \\
1 & \ddots & & \vdots \\
\vdots & & \ddots & 1 \\
1 & \cdots & 1 & \mathrm{~m}+2
\end{array}\right) \times \operatorname{det}\left(\begin{array}{cccc}
\mathrm{n}+2 & 1 & \ldots & 1 \\
1 & \mathrm{n}+2 & & \vdots \\
\vdots & & \ddots & 1 \\
1 & \ldots & 1 & \mathrm{n}+2
\end{array}\right)
\end{aligned}
$$

Applying lemma 13 with $\mathrm{x}=\mathrm{m}+2$ and $\mathrm{x}=\mathrm{n}+2$, respectively, we have:

$$
\tau\left(\mathrm{K}_{1}+\mathrm{K}_{\mathrm{n}, \mathrm{m}}\right)=(\mathrm{n}+\mathrm{m}+1) \times(\mathrm{n}+1)^{\mathrm{m}-1} \times(\mathrm{m}+1)^{\mathrm{n}-1}
$$

Specially, $\tau\left(K_{1}+K_{n, n}\right)=(2 n+1) \times(n+1)^{2 n-2}$.

Corollary24: $\tau\left(K_{n, n} \circ e\right)=(2 n-1) n^{2 n-4}=\tau\left(K_{1}+K_{n-1, n-1}\right)$.

Theorem 25: $\tau\left(K_{2} \times K_{n, n}\right)=2 n^{2 n-2} \times(n+1) \times(n+2)^{2 n-2}$.

Proof: Applying lemma 2, we have:

$$
\tau\left(K_{2} \times K_{n, n}\right)=\frac{1}{(4 n)^{2}} \operatorname{det}(4 n I-\bar{D}+\bar{A})=\frac{1}{(4 n)^{2}} \operatorname{det}
$$$$
\left(\begin{array}{cccccccccccccc}
\mathrm{n}+2 & 0 & 1 & 0 & 1 & \cdots & \cdots & 0 & 1 & 1 & \cdots & \cdots & \cdots & 1 \\
0 & \mathrm{n}+2 & 0 & 1 & 0 & \ddots & \ddots & 1 & 0 & 1 & \ddots & \ddots & \ddots & \vdots \\
1 & 0 & \mathrm{n}+2 & 0 & 1 & 0 & \ddots & \vdots & 1 & \ddots & \ddots & \ddots & \ddots & \vdots \\
0 & 1 & 0 & \ddots & 0 & \ddots & \ddots & \vdots & \vdots & \ddots & \ddots & \ddots & \ddots & \vdots \\
1 & \cdots & \ddots & \ddots & \ddots & 1 & 1 & \vdots & \vdots & \ddots & \ddots & \ddots & \ddots & \vdots \\
\vdots & \cdots & \cdots & 1 & 0 & \mathrm{n}+2 & 0 & \vdots & \vdots & \ddots & \ddots & \ddots & 0 & 1 \\
\vdots & \cdots & \cdots & \vdots & 1 & 0 & \mathrm{n}+2 & 1 & \cdots & \cdots & \cdots & \cdots & 1 & 0 \\
0 & 1 & \cdots & \cdots & \cdots & \cdots & 1 & \mathrm{n}+2 & 0 & 1 & 0 & 1 & \cdots & \cdots \\
1 & 0 & \ddots & \ddots & \ddots & \ddots & \vdots & 0 & \mathrm{n}+2 & 0 & \ddots & \ddots & \ddots & \vdots \\
\vdots & 1 & \ddots & \ddots & \ddots & \ddots & \vdots & 1 & 0 & \mathrm{n}+2 & \ddots & \ddots & \ddots & \vdots \\
\vdots & \ddots & \ddots & \ddots & \ddots & \ddots & \vdots & 0 & 1 & 0 & \ddots & \ddots & \ddots & \vdots \\
\vdots & \ddots & \ddots & \ddots & \ddots & \ddots & \vdots & \vdots & \ddots & \ddots & \ddots & \ddots & \ddots & 1 \\
\vdots & \ddots & \ddots & \ddots & \ddots & 0 & 1 & \vdots & \ddots & \ddots & \ddots & \ddots & \mathrm{n}+2 & 0 \\
1 & \ddots & \ddots & \ddots & \ddots & 1 & 0 & \vdots & \ddots & \ddots & \ddots & \ddots & 0 & \mathrm{n}+2
\end{array}\right)
$$

Using lemma14, we get:

$$
\tau\left(\mathrm{K}_{2} \times \mathrm{K}_{\mathrm{n}, \mathrm{n}}\right)=\frac{1}{(4 \mathrm{n})^{2}} \operatorname{det}\left(\begin{array}{ccccccc}
\mathrm{n}+2 & 1 & 2 & 1 & 2 & \cdots & \cdots \\
1 & \mathrm{n}+2 & 1 & \ddots & \ddots & \ddots & \ddots \\
2 & 1 & \mathrm{n}+2 & \ddots & 2 & \ddots & \ddots \\
1 & \ddots & \ddots & \ddots & \ddots & \ddots & \ddots \\
2 & \ddots & \ddots & \ddots & \ddots & \ddots & \ddots \\
\vdots & \ddots & \ddots & \ddots & \ddots & \mathrm{n}+2 & 1 \\
\vdots & \ddots & \ddots & \ddots & \ddots & 1 & \mathrm{n}+2
\end{array}\right) \times
$$

Straightforard induction using properties of determinants.

We have:

$$
\begin{aligned}
\tau\left(\mathrm{K}_{2} \times \mathrm{K}_{\mathrm{n}, \mathrm{n}}\right) & =\frac{1}{(4 \mathrm{n})^{2}} \times 8 \mathrm{n}^{2 \mathrm{n}} \times 4(\mathrm{n}+1)(\mathrm{n}+2)^{2 \mathrm{n}-2} \\
& =2 \mathrm{n}^{2 \mathrm{n}-2} \times(\mathrm{n}+1) \times(\mathrm{n}+2)^{2 \mathrm{n}-2}
\end{aligned}
$$

Theorem 26: $\tau\left(K_{2} \circ K_{n, n}\right)=2^{4 n-2} \times n^{2 n-2} \times(n+1)^{2 n}$.

Proof: Apply lemma 2, we have:

$$
\tau\left(K_{2} \circ K_{n, n}\right)=\frac{1}{(4 n)^{2}} \operatorname{det}(4 n I-\bar{D}+\bar{A})=\frac{1}{(4 n)^{2}} \operatorname{det}
$$

$$
\left(\begin{array}{cccccccccccccc}
2 \mathrm{n}+2 & 0 & 1 & 0 & 1 & \cdots & \cdots & 0 & 0 & 1 & 0 & 1 & \cdots & \cdots \\
0 & 2 \mathrm{n}+2 & 0 & 1 & 0 & \ddots & \ddots & 0 & 0 & 0 & 1 & 0 & 1 & \ddots \\
1 & 0 & 2 \mathrm{n}+2 & 0 & 1 & 0 & \ddots & \vdots & 1 & \ddots & \ddots & \ddots & \ddots & \ddots \\
0 & 1 & 0 & \ddots & 0 & \ddots & \ddots & 0 & 0 & \ddots & \ddots & \ddots & \ddots & \ddots \\
1 & \cdots & \ddots & \ddots & \ddots & 1 & 1 & 1 & \vdots & \ddots & \ddots & \ddots & \ddots & \ddots \\
\vdots & \cdots & \cdots & 1 & 0 & 2 \mathrm{n}+2 & 0 & \vdots & \vdots & \ddots & \ddots & \ddots & \ddots & \ddots \\
\vdots & \cdots & \cdots & \vdots & 1 & 0 & 2 \mathrm{n}+2 & 1 & \vdots & \ddots & \ddots & \ddots & \ddots & \ddots \\
0 & 0 & 1 & 0 & 1 & \cdots & \cdots & 2 \mathrm{n}+2 & 0 & 1 & 0 & 1 & \cdots & \cdots \\
0 & 0 & 0 & 1 & 0 & \ddots & \cdots & 0 & 2 \mathrm{n}+2 & 0 & \ddots & \ddots & \ddots & \ddots \\
1 & 0 & 0 & 0 & \ddots & \ddots & \ddots & 1 & 0 & 2 \mathrm{n}+2 & \ddots & \ddots & \ddots & \ddots \\
0 & 1 & \ddots & \ddots & \ddots & \ddots & \ddots & 0 & \ddots & 0 & \ddots & \ddots & \ddots & \ddots \\
1 & 0 & \ddots & \ddots & \ddots & \ddots & \ddots & \ddots & \ddots & \ddots & \ddots & \ddots & \ddots & \ddots \\
0 & 1 & \ddots & \ddots & \ddots & \ddots & \ddots & \ddots & \ddots & \ddots & \ddots & 2 \mathrm{n}+2 & 0 \\
\vdots & \ddots & \ddots & \ddots & \ddots & \ddots & \ddots & \ddots & \ddots & \ddots & \ddots & 0 & 2 \mathrm{n}+2
\end{array}\right)
$$

Using lemma14, we get:

$$
\tau\left(\mathrm{K}_{2} \circ \mathrm{K}_{\mathrm{n}, \mathrm{n}}\right)=\frac{1}{(4 \mathrm{n})^{2}} \operatorname{det}\left(\begin{array}{ccccccc}
2 \mathrm{n}+2 & 0 & 2 & 0 & 2 & \cdots & \cdots \\
0 & 2 \mathrm{n}+2 & 0 & \ddots & \ddots & \ddots & \ddots \\
2 & 0 & 2 \mathrm{n}+2 & \ddots & 2 & \ddots & \ddots \\
0 & \ddots & \ddots & \ddots & \ddots & \ddots & \ddots \\
2 & \ddots & \ddots & \ddots & \ddots & \ddots & \ddots \\
\vdots & \ddots & \ddots & \ddots & \ddots & 2 \mathrm{n}+2 & 0 \\
\vdots & \ddots & \ddots & \ddots & \ddots & 0 & 2 \mathrm{n}+2
\end{array}\right) \times
$$

Straightforward induction using properties of determinants. 
We have:

$\tau\left(\mathrm{K}_{2} \circ \mathrm{K}_{\mathrm{n}, \mathrm{n}}\right)=2^{4 \mathrm{n}-2} \times \mathrm{n}^{2 \mathrm{n}-2} \times(\mathrm{n}+1)^{2 \mathrm{n}}$

Theorem 27: $\tau\left(\mathrm{K}_{2} \odot \mathrm{K}_{\mathrm{n}, \mathrm{n}}\right)=(\mathrm{n}+1)^{4 \mathrm{n}-4} \times(2 \mathrm{n}+1)^{2}$.

Proof: Applying lemma 2, we have:

$$
\begin{aligned}
& \tau\left(K_{2} \odot K_{n, n}\right)=\frac{1}{4(2 n+1)^{2}} \operatorname{det}((4 n+2) I-\bar{D}+\bar{A})=\frac{1}{4(2 n+1)^{2}} \operatorname{det} \\
& \left(\begin{array}{cccccccccccccc}
2 \mathrm{n}+2 & 0 & 0 & \ldots & \ldots & \ldots & 0 & 0 & 1 & 1 & \cdots & \cdots & \cdots & 1 \\
0 & \mathrm{n}+2 & 0 & 1 & 0 & \ddots & \vdots & 1 & 1 & \ddots & \ddots & \ddots & \ddots & \vdots \\
0 & 0 & \mathrm{n}+2 & 0 & 1 & \ddots & \vdots & 1 & \ddots & \ddots & \ddots & \ddots & \ddots & \vdots \\
\vdots & 1 & 0 & \ddots & 0 & \ddots & \vdots & \vdots & \ddots & \ddots & \ddots & \ddots & \ddots & \vdots \\
\vdots & 0 & \ddots & \ddots & \ddots & \ddots & 1 & \vdots & \ddots & \ddots & \ddots & \ddots & \ddots & \vdots \\
\vdots & \vdots & \ddots & \ddots & 0 & \mathrm{n}+2 & 0 & \vdots & \ddots & \ddots & \ddots & \ddots & \ddots & \vdots \\
0 & \vdots & \ddots & \ddots & 1 & 0 & \mathrm{n}+2 & 1 & \ldots & \ldots & \ldots & \ldots & \ddots & 1 \\
0 & 1 & 1 & \ldots & \ldots & \ldots & 1 & 2 \mathrm{n}+2 & 0 & 0 & 0 & \ldots & \cdots & 0 \\
1 & 1 & \ldots & \ddots & \ddots & \ddots & \vdots & 0 & \mathrm{n}+2 & 0 & 1 & 0 & \cdots & \ldots \\
1 & \ddots & \ddots & \ddots & \ddots & \ddots & \vdots & 0 & 0 & \mathrm{n}+2 & 0 & \ddots & \ddots & \ddots \\
\vdots & \ddots & \ddots & \ddots & \ddots & \ddots & \vdots & \vdots & 1 & 0 & \ddots & \ddots & \ddots & \ddots \\
\vdots & \ddots & \ddots & \ddots & \ddots & \ddots & \vdots & \vdots & \vdots & \ddots & \ddots & \ddots & \ddots & \ddots \\
\vdots & \ddots & \ddots & \ddots & \ddots & \ddots & \vdots & \vdots & \vdots & \ddots & \ddots & \ddots & \mathrm{n}+2 & 0 \\
1 & \ldots & \ldots & \ldots & \ldots & \ldots & 1 & 0 & \vdots & \ddots & \ddots & 1 & 0 & \mathrm{n}+2
\end{array}\right)
\end{aligned}
$$

Using lemma14, we get:

$$
\tau\left(\mathrm{K}_{2} \odot \mathrm{K}_{\mathrm{n}, \mathrm{n}}\right)=\frac{1}{4(2 \mathrm{n}+1)^{2}} \operatorname{det}\left(\begin{array}{ccccccc}
2 \mathrm{n}+2 & 1 & 1 & \ldots & \ldots & \cdots & 1 \\
1 & \mathrm{n}+3 & 1 & 2 & 1 & \cdots & \cdots \\
1 & 1 & \mathrm{n}+3 & \ddots & \ddots & \ddots & \ddots \\
\vdots & 2 & \ddots & \ddots & \ddots & \ddots & \ddots \\
\vdots & 1 & \ddots & \ddots & \ddots & \ddots & \ddots \\
\vdots & \vdots & \ddots & \ddots & \ddots & \mathrm{n}+3 & 1 \\
1 & \vdots & \ddots & \ddots & \ddots & 1 & \mathrm{n}+3
\end{array}\right) \times
$$

$$
\operatorname{det}\left(\begin{array}{ccccccc}
2 \mathrm{n}+2 & -1 & -1 & \cdots & \cdots & \cdots & -1 \\
-1 & \mathrm{n}+1 & -1 & 0 & -1 & \cdots & \cdots \\
-1 & -1 & \mathrm{n}+1 & \ddots & \ddots & \ddots & \ddots \\
\vdots & 0 & \ddots & \ddots & \ddots & \ddots & \ddots \\
\vdots & \vdots & \ddots & \ddots & \ddots & \ddots & \ddots \\
\vdots & \vdots & \ddots & \ddots & \ddots & \mathrm{n}+1 & -1 \\
-1 & \vdots & \cdots & \cdots & 0 & -1 & \mathrm{n}+1
\end{array}\right)
$$

Straightforward induction using properties of determinants.

We have:

$$
\begin{gathered}
\tau\left(K_{2} \odot K_{n, n}\right)=\frac{1}{4(2 n+1)^{2}} \times 2(n+1)^{2 n-2} \times(2 n+1)^{3} \\
\times 2(n+1)^{2 n-2} \times(2 n+1)=(n+1)^{4 n-4} \times(2 n+1)^{2}
\end{gathered}
$$

\section{ACKNOWLEDGMENT}

The researcher is deeply indebted to the team of work at deanship of scientific research at Taibah university, Al-Madinah Al-Munawwarah, K.S.A for their continuous helps and the encouragement us to finalize this study.

\section{REFERENCES}

Austin,T.L., 1960. The enumeration of point labeled chromatic graphs and trees. Canad. J. Math., 12: 535-545.

Balakrishnan, R. and K. Ranganathan, 2000. A Textbook of Graph Theory. 1st Edn., Springer, New York, ISBN-10: 0387988599 pp: 227.

Bermond, J.C., F. Comellas and D.F. Hsu, 1995. Distributed loop computer networks: A survey. J. Parallel Distrib. Comput., 24: 2-10.

Biggs, N., 1993. Algebraic Graph Theory. 2nd Edn., Cambridge Univ. Press, Cambridge, ISBN-10: 0521458978 pp: 205.

Brown, T.J.N., R.B. Mallion, P. Pollak and A. Roth, 1996. Some methods for counting the spanning trees in labelled molecular graphs, examined in relation to certain fullerenes. Discreate Applied Math., 67: 51-66. DOI: 1016/0166218X(96)85158-4

Cayley, G.A., 1889. A Theorm on Trees. Quart. J. Math., 23: 276-378.

Clark, L., 2003. On the enumeration of multipartite spanning trees of the complete graph. Bull. ICA., 38: 50-60.

Colbourn, C.J., 1987. The Combinatorics of Network Reliability. 1st Edn., Oxford University Press, New York, ISBN-10: 0195049209 pp: 160.

Cvetkovic, D.M., M. Doob and H. Sachs, 1980. Spectra of Graphs: Theory and Application. Academic Press, New York, ISBN-10: 0121951502 pp: 368.

Egecioglu, O. and J.B. Remmel, 1994. A bijection for spanning trees of complete multipartite graphs. University of California.

Lewis, R.P., 1999. The number of spanning trees of a complete multipartite graph. Discrete Math., 197198: 537-541. DOI: 10.1016/S0012365X(99)90111-5

Myrvold, W., K.H. Cheung, L.B. Page and J.E. Perry, 1991. Uniformly-most reliable networks do not always exist. Networks, 21: 417-419. DOI: $10.1002 /$ net.3230210404 
Petingi, L., F. Boesch and C. Suffel, 1998. On the characterization of graphs with maximum number of spanning trees. Discrete Math., 179: 155-166. DOI: 10.1016/S0012-365X(97)00034-4

Porter, T.D., 2004. Generating the list of spanning trees $\mathrm{K}_{\mathrm{p}, \mathrm{q}}$. J. Comb. Comput., 50: 17-32.
Wilson, R.J. and J.J. Watkins, 1990. Graphs: An Introductory Approach : A First Course in Discrete Mathematics. 1st Edn., Wiley, New York, ISBN10: 0471615544 pp: 340. 